work. It is to be placed on the seat of a chair, and the patient sits on it with the higher side towards the convexity of the spine (Fig. 4), thus by degrees forcing the column to assume a straight line or a contrary curve. This cannot, however, be used in the first part of the case for very long at a time, but rather for several short periods during the

FIG. 4.

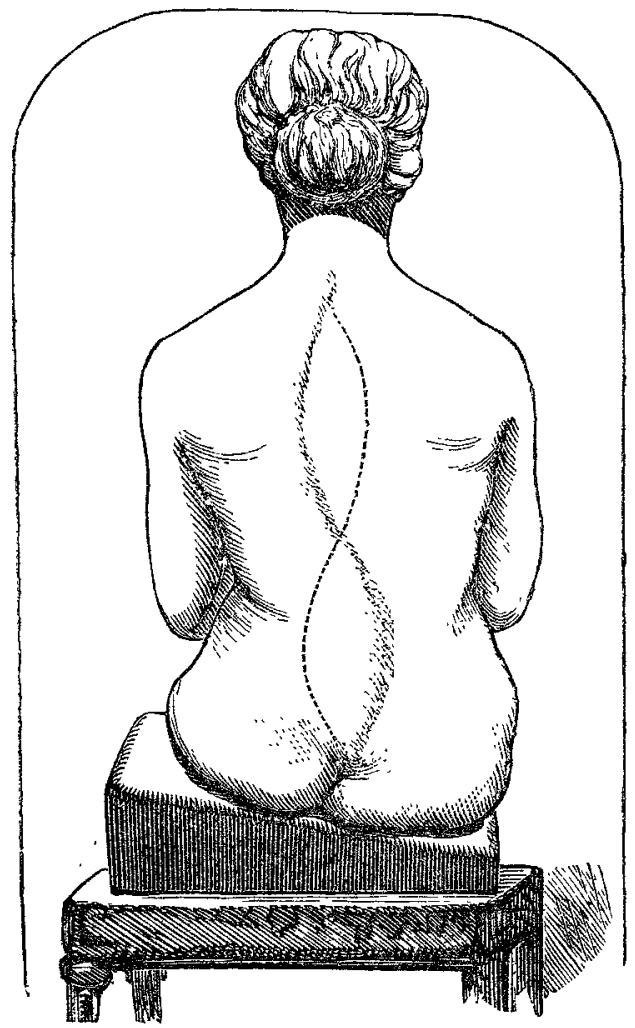

The dotted line represents the morbid curve, the shaded line that which the artificial pelvic obliquity tends to produce.

day. Hence it is convenient to let the patient sit on it during meals. For quite young subjects with commencing curves these methods often suffice.

But in more developed cases some power which shall draw the convexity over to the concave side is required,
FIG. 5.

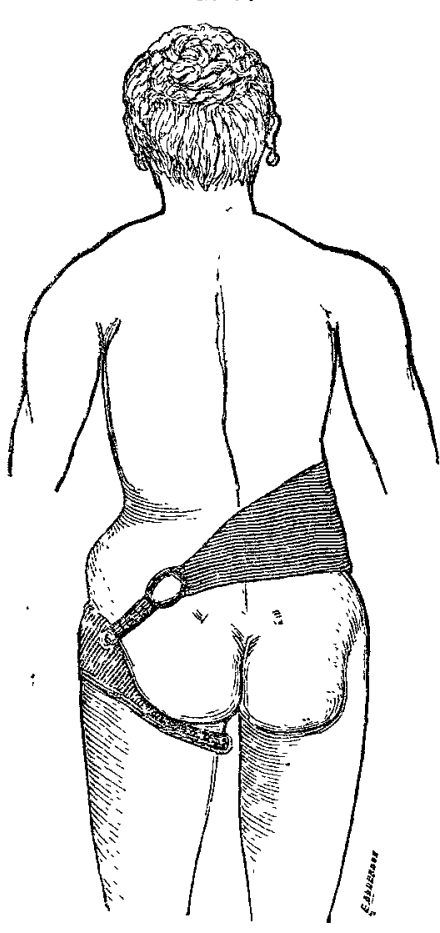
at an acute angle with the direction of the leg strap. Rarely
is any other device than those above named required, but in really severe cases two others may be added.

The lateral swing is a mechanism that I used for some years before giving any account of it. (Fig. 6.) Dr. Busch and I seem to have published it simultaneously. I chiefly use it in dorsal curvature, but also occasionally in lumbar. It is fourteen inches long by six broad and ten high ; it should therefore be kept at the bedside, and when the patient retires for the night she lies in this for about twenty minutes, so that the convex side of $+1 \theta$ spine is slung up off the hed by the broad webbing, and must curve in the contrary direction. Again, in the morning, quite early, the maid or nurse can hand this into the bed, and the patient can lie and take an additional sleep in it.

The loop girth is simply a piece of broad webbing about four feet long, fastened by each end to a staple in the wall a little above the level of the patient's waist; she stands with the concave side towards the wall; the convex side is caught in the loop, the foot on that side being supported by a blocks from one to two inches high. When the girth is found to be properly secure, she places her clasped hands on ber hear and slowly bends over; then rises again and repeats this manouvre several times, gradually increasing the number, as also the rapidity, of the movements, as custom renders them easier. Very few, if any, lumbar curves resist these combined remedies.

In my next paper the more difficult subject of dorsal curvature will be discussed.

\section{CANCER OF BRONCHIAL GLANDS AND LUNG. ${ }^{1}$}

Bx M. A. BOYD, M.C.P. IREL., PHYSICIAN TO MATER MISERTCORDIE HOSPYTAL, DUBLIY.

IN bringing forward the subject of cancer of the bronchial glands and lung $I$ am not calling attention to any new subject, but $I$ think I may venture to assert it is a subject the importance of which cannot be exaggerated, and the difficulties in the diagnosis of which all physicians must admit. From the enormous amount of adenoid tissue within the thorax, and the well-known affinity cancer has for that structure, we should reason a priori that intra thoracic cancer should be of not unfrequent occurrence, and from the marked similarity between it and phthisis, especially the so-called senile variety of phthisis, in many of its symptoms, the existence of the disease is, I am convinced, frequently overlooked. The characteristic sym. ptoms that are said to indicate intra-thoracic cancer of either the mediastinum or glands, such as fixed pain in the chest, red-currant-jelly expectoration, and enlargement of cervical glands, are not present in a fourth of the cases, while symptoms that indicate phtbisis are present in the great majority of them, and in some the presence of cancer is not even suspected until widespread infiltration of either the lung, pleura, or pericardium reveals its existence. Enlargement of one or more cervical glands when present, with symptoms of a tumour within the thorax, not aneurysmal, would be to us conclusive of the presence of cancer, but this evidence is more often wanting than not. The same remarks apply to the existence of pain, which is seldom present to any extent until symptoms of pressure on the large nervous trunks occur, and this is generally so late a symptom that all doubt as to the nature of the case is set at rest before pain becomes a prominent symptom. Red-currant-jelly expectoration is in my experience a very rare symptom. I am aware that in these assertions I am not in keeping with the generally received opinions on the subject, but unfortunately (he front one. When the belance is arranged aright, the whole sits very comfortably, and does not show under the garments. A very small percentage of patients feel for a day or two the strap round the leg; they very soon lose the sensation, for there is really no tension on it, the force of the loin part acting
1 Read before the Academy of Medicine in Ireland, May 28th, 1886. 
every-day experience proves to us that generally received opinions are often erroneous, and we find often when it is too late that all the statements in standard works are not invariably to be relied on; especially so is the generally accepted one that cancer of the lung or mediastinum is generally secondary to cancer in other organs, so that our conclusions are unsatisfactory even where symptoms arouse our suspicion, and we look for its presence elsewheregenerally not to find it.

Cancer of the bronchial glands and lung, in my experience, nearly always begins as primary cancer in these situations, and very rarely as secondary infiltration from other parts, and where it is secondary there is generally well-marked engagement of the lymphatic system in other situations before it becomes intra-thoracic. The intra-thoracic situstions where malignant disease is met are two-namely, the bronchial glands and the lung, and anterior mediastinum. The former situation is from a clinical point of view the most important, as malignant disease in these structures, simulates phthisis in a subacute form so closely that its differential diagnosis in some cases is almost impossible. From observation of a good number of cases I am conwinced this is so, and a case of malignant deposit in the bronchial glande, infiltrating the lung, ending in ulceration and in the formation of cavities, is frequently set down as one of hopeless phthisis, a post-mortem on which would be of no interest, and all record of the frequency of the disease is in consequence entirely lost. With mediastinal cancer such an error is not so likely to occur, as, like other intra-thoracic growths, such as aneurysm, the symptoms are more the sesult of the pressure it exercises on the important vascular and nervous structures around it, and which attract attention long before infiltration of the lung and general cachexia show themselves. Added to this, the growth is more superficial and more easy to be recognised. With the former variety lung symptoms predominate; with the latter cardiac and vascular ones. Hæmoptosis appearing early, and being constant in its occurrence, would seem to be the most reliable sign, and was present in more than half of thirty-nine cases recorded by Dr. Risdon Bennett in his Lumleian Lectures on Intrathoracic Cancer, and Dr. Walshe speaks of it as having occurred at some period in nearly all of his cases. When persistently present it affords evidence of the situation of the growth in the bronchial glands or in the lung itself, with pressure on the bronchial veins, as distinguished from mediastinal cancer, which latter, as Dr. Cockle remarks in his work, may afford no evidence of its existence until pressure symptoms, or infiltration of the surrounding parts, especially the pericardium or large vessels, indicate the presence of a tumour within the thorax.

I will now confine my remarks principally to the symptoms that indicate cancerous engagement of the bronchial glands and lung and their similarity to phthisis, and afterwards to those that indicate engagement of the mediastinum. have mentioned hæmoptosis as a constant and oft-recurring symptom. Next in importance, as pointed out by Dr. Rossbach, would seem to be early congestion of the larynx, with aphonia, and without paralysis of the rocal cords, which latter symptom I will allude to afterwards as conclusive of mediastinal cancer. Spasmodic cough, with very little expectoration in proportion,as indicating early irritation of the bronchial nerves, frequently accompanied by dyspnoea and vomiting, is another early symptom. Next, dulness on percussion over some portion of the chest anteriorly; but this is not always present in the early stages of cancer of the bronchial glands, though it is the earliest symptom in mediastinal cancer. Feeble respiratory sounds on one side, with bronchial râles, tubular breathing, prolongation of the expiratory sounds, and symptoms indicating pressure on and narrowing of the bronchial tubes, are frequently present. Difficulty in swallowing from pressure on the œsophagus, with cough and vomiting excited by the effort, and a venous hum over the upper portion of the sternum when the head is thrown back, as pointed out by Dr. Eustace Smith when describing the symptoms indicating enlarged bronchial glands, might be placed next in order. Pleural effusion into either one or the other side, containing blood when withdrawn by aspiration. This latter phenomenon is said to be characteristic of intra-thoracic cancer. though microscopic examination of the pleural fluid as well as of the sputa in these cases generally gives negative results as regards the presence of the characteristic cells. If we add to many of the above symptoms in advanced cases and in old people muco-purulent expectoration, emaciation, night sweate, and a moderately high temperature, we liave a chain of phenomena that resemble phthisis.

When cancer begins in the anterior mediastinum there may be no cough whatever in the early stage, but there is marked retro-sternal dulness, with sense of resistance, and generally retro-sternal pain. Here pressure symptoms begin to show themselves early, as indicated by displacement of the heart, fulness of the veins in the neck, with cyanosis and cedema of the face and upper extremities, and luter on dyspncea, with stridulous breathing, aphonia, and paralysis of one or other of the vocal cords, as the vagus or recurrent laryngeal respectively get pressed on. Paralysis of one or other of the vocal cords, with symptoms of a tumour within the thorax in the absence of aneurysm, is regarded by Dr. Burney Yeo as absolutely diagnostic of malignant disease. Though the symptoms of cancer of the bronchial glands resemble phthisis very closely, we shall find in the signs occasionally feat ures that raise doubts in our minds as to their being phthisical ones. The dulness on percussion is generally not apical, but lower down over the lung, and is elicited posteriorly opposite to the same situation in front, and is generally more extensive than in phthisis, when the disease is of some duration. There is also generally an absence of all crepitus, though bronchial râles and feeble in spiratory sounds, with prolongation of expiration, are present. When the bronchial glands are much enlarged and compress the bronchi, vocal fremitus and vocal resonance are both absent; and absence of all breath sounds with collapse or consolidation of the lung generally present a condition you will not find in phthisis, no matter how extensive the consolidation. Though many of the above symptoms are present in a good number of the cases of primary cancer of the bronchial glands, in some one of the symptoms will be most marked, and the first of the following cases illustrates the prominence of pleural effusion over all other symptoms.

John G-, aged thirty-eight, a bottle-blower by trade, was admitted into the Mater Misericordiæ Hospital on the 27 th of July last, suffering from cough, dyspnoea, lividity of the face, and slightly bloody expectoration. He never had syphilis, rheumatism, or any other illness, though be drank hard at times, and was able to attend to his work, in which he was constantly exposed to draughts for a period of fourteen hours at a time. There was no history of malignant disease in his family. Three weeks before admission, while engaged at his occupation, he caught cold, which showed itself by prolonged rigor, sickness of stomach, and headache with feverishness; he also felt pain of a catching character in the right mammary region. He was confined to bed for a few days with these symptoms; but after this time was able to attend to his work again, experiencing only slight pain in the right side, cough, and shortness of breath. For a week before admission these symptoms became worse, and on admission he presented the following:Lividity of the lips and face, with puffiness of the eyelids and prominence of the veins on the front of the thorax and also in the neck; no enlarged gland to be seen. Ilis voice was hoarse and aphonic, and there was urgent dyspnca, especially on exertion, with cough and bronchial fremitus over the chest. The patient seemed in no way emaciated, and did not suffer from night sweats. There was absolute dulness on percussion over the entire aspect of the right chest, except for an inch or two under the clavicle, where it was tympanitic; and behind, the dulness reached as high as the centre of the scapula. In front it encroached across the upper portion of the sternum to the extent of an inch or two beyond its left border, and here the dulness gave great sense of resistance. There was feeble inspiration through the right lung, with bronchial râles in botb; and vocal fremitus and vocal resonance were both absent on the right side. The heart was feeble in its action, but not displaced, and no murmur was present either in it or the great vessels. From enlarged measurements of the right side and the above symptoms I considered there was fluid in the pleura, though I could not explain the symptoms of venous pressure from this alone, and could only conjecture there was a tumour within the thorax also. I aspirated the right pleura on the day after his admission, removing nine pints of fluid, the latter portion of which was bloody. After removal of the fluid the dulness was more distinct over the upper portion of the sternum, and could be defined. His dyspnoea was considerably relieved, and more air seemed to enter the 
right lung, cyanosis of the head and face, however, continuing, and the veins on the thorax showing more prominently. A week later his right arm and face became very swollen, and, recurring dulness over the right side indicating fresh effusion, I again tapped the pleura and removed six pints of bloody fluid. Some days after the second tapping his temperature rose, his dyspnoea increased, and duiness at the base of the left lung showed itself. Suppression of urine with delirium set in, and he died on August 18th. The post-mortem examination showed a large mass of cancer, both hard and medullary, infiltrating the bronchial glands and lung on the right side, beginning at the bifurcation of the trachea, and involving in its course the pleura, which was thickened, lymphy, and contained a quantity of bloody serum, and infiltrated the pericardium over the auricle, the superior cava of which it compressed. The left lung was congested throughout, and solidified around its main bronchi trom recent inflammation.

The following case, in which symptoms of phthisis with difflculty in swallowing were the most prominent features, shows a different' train of symptoms :-

James $\mathrm{McC}$ _ a waiter, aged thirty-seven, was admitted into the Mater Misericordi $\infty$ Hospital on Sept. 22nd last, complaining of cough, with muco-purulent expectoration mixed with blood, night sweats, emaciation, and symptoms of stricture of the œsophagus. The patient gave a good family history; there bad been no phthisis on either side. He drank freely, taking as many as eight or ten glasses of whisky daily, undiluted. He had never suffered from any illness until six months before admission, when he noticed a difficulty in swallowing his food, accompanied by cough and vomiting, the food being arrested at a spot which he referred to the centre of the sternum. There was no pain at the place of constriction, and no sense of pressure or dyspnoa. He coughed up frequently considerable quantities of blood, bringing up nearly half a pint six weeks before admission, occasionally being jam-tinted or prune-juiced in colour. On examination the heart was found healthy; no murmur. There was no enlargement of the cervical glands. The throat was congested and the voice aphonic. Dulness was elicited over the upper portion of the sternum, extending to the subclavian space on the right side, with tubular breathing and bronchial râles, but no crepitus. There was no dulness posteriorly, the breath sounds being normal here. An csophageal tube of the smallest size could be passed, and the stricture only admitted the next size up to the time of his death; all attempts to pass a larger one failed. After catheterisation liquid nourishment could be taken, but it always induced cough at first, ending in vomiting. The temperature throughout varied, rising occasionally from normal to $101 \cdot 30^{\circ}$. His emaciation and night sweats increased, the tube being passed daily to allow of nourishment being taken until Oct. 17 th, when the dulness over the upper portion of the sternum and right apex showed a marked increase, and on the 21st no tube could be passed, the attempts to do so causing considerable pain. Pulmonary congestion set in, and the patient died on Oct. 28th. I regret that in this case no post-mortem examination was allowed, as we should no doubt have found enlarged cancerous bronchial glands pressing on and constricting the oesophagus springing from the fork of the trachea, which would seem to be the most usual site for cancer in this situation to begin.

The next case, a washerwoman, aged fifty, was admitted into my ward in the hospital with feverish symptoms, pain in the left side, dyspnoea, wasting cough, and mucopurulent expectoration mixed with blood, which was constantly present. The patient was seen in my absence by the assistant physician, who diagnosed pleural effusion of the left side, with dulness on percussion at base, absence of vocal fremitus, and resonance. Though no dulness existed in the upper portion of the lung, there was complete absence of all breath sounds here, which seemed rather difficult to explain. The woman showed marked hectic flush, and dyspnoea existed out of all proportion to the pleural effusion. Her illness began about three months previously with pain in the left side, a cough that had existed for some months before, with blood-spitting; but the symptoms were not urgent until a few weeks before admission. There was dulness in front behind the upper portion of the sternum, extending to and becoming continuous with cardiac dulness, but the percussion over both apices was normally clear. The respiration was puerile at the right apex and over the right lung; but there was complete absence of all respiratory sounds over the left apex. The dyspnœa and orthopnœa were most troublesome, and the temperature remained high up to the time of her death, which occurred three weeks after her admission. The post-. mortem examination showed dark-coloured serum in the left pleural cavity to the extent of two or three pints. The pleura and lung were adherent at its upper and anterior aspect, with a thickened mass of hard bronchial glands surrounding the root of the left lung, compressing the bronchit and bronchial veins. The lung itself was contracted to half its size, and puckered here and there from emphysematous lobules, and the upper lobes studded in places with cancerous. nodules, both medullary and scirrhous, which spread in a. radiating manner from its root; between these the lung was solidified and non-crepitant.

I may mention that in both these cases microscopic sections of the deposit showed the typical cells. And in the first two cases, though aphonia and congestion of the larynx. were present, there was no paralysis of the vocal cords evident. I need not here allude to secondary cancer occurring in these situations, my object being to show that primary cancer is of the two much more frequent, and that in advanced cases it is likely to be confounded with phthisis.

The general conclusions I have come to with regard to the disease are as follows: That primary cancer of the thorax is of not unfrequent occurrence, generally appearing in hard drinkers, and the symptoms of it are developed apparently after catching cold. That it is to be found in two situations within the thorax-namely, the bronchial glands and the anterior mediastinum; and is of the mixed variety, scirrhous and encephaloid. That persistent blood-spitting is a very usual symptom in its growth, and indicates infiltration of the lung and pressure on the bronchial veins; and that the so-called characteristic red-current-jelly expectoration is a rare symptom. That fixed pain in the thorax is not al ways present, and when it is, it is more usual in the mediastinal form of the disease. That the disease is generally accompanied by aphonia and congestion of the larynx in its early stages, and by paralysis of one or other of the vocal cords in a later stage. That when in the bronchial glands it is invariably accompanied by symptoms of pressure on either one or other of the bronchi, or on the cesophagus, causing symptoms of stricture. That pleural effusion containing blood is very frequent on the side affected, and in its growth forwards from the root of the lung it involves the pericardium, and causes pressure on the superior cava or pulmonary veins. That when in the bronchial glands its later stages may simulate advanced phthisis very closely. That it may be occasionally distinguished from phthisis by its pressure symptoms on the bronchi, its dulness being more central and more extensive, with absence of crepitus and more urgent dyspncea, while no bacilli are found in the expectoration. That malignant intra-thoracic disease is generally fatal in from two months to six, though Dr. Walshe gives the average duration as from three months to thirteen. In the cases I have just mentioned the disease was fatal in each case within seven months. I need not here allude to the difficulty in distinguishing between intra-thoracic malignant disease and aneurysm, where no murmur is present and pressure symptoms are the most prominent feature in the case. The difficulties of forming a differential diagnosis in such cases are indeed great-nay, sometimes almost impossible,-and we have mainly to rely on the evidence afforded by arterial disease elsewhere to assist us.

\section{MOLLUSCUM FIBROSUM AND LARGE PENDULOUS FIBROMA;}

REMOVAL OF GROWTH; RECOVERY.

BY HENRY A. LEDIARD, M.D. EdIN., F.R.C.S. ENG., SURGEON TO THE CUMBERLAND INFIRMARY.

JонN C-_, aged twenty-nine, labourer in a timber yard was admitted into the Cumberland Infirmary on March 17 th, 1886. The patient has never been what is called "quick," and his head has always been very large in proportion to his body. He has had none but ordinary illnesses, with the exception of bronchitis, from which he suffered for the previous three months. He has had from birth a tumour over the left hip, which continued to grow till he was twentyfour, when it ceased growing. In addition to this, he has developed numerous small tumours on various parts of the 